\title{
Quantificação de informação na teoria de Bayes em escalas temporais
}

\author{
Igor de S. Barbalho Lucas Ragiotto \\ Depto de Matemática, FEIS, UNESP \\ 15385-000, Ilha Solteira, SP \\ E-mails: igorsouza4@gmail.com lucasragiotto@gmail.com \\ Berenice C. Damasceno Luciano Barbanti \\ Depto. de Matemática, FEIS, UNESP \\ 15385-000, Ilha Solteira, SP \\ E-mails: berenice@mat.feis.unesp.br barbanti@mat.feis.unesp.br
}

\section{RESUMO}

\section{Medida de "surpresa"}

A teoria da informação permeia todos os campos da ciência. Um dos primeiros problemas que a teoria pressupõe é como quantificar a informação de um conjunto de dados.

Shannon em 1948 quantificou a informação de um conjunto de dados C, dentro de uma mensagem $M$, como sendo $\log _{2} \mathrm{P}_{\mathrm{M}}(\mathrm{C})$.

Na década de 2000 Baldi e Itti [2], diante dos conceitos envolvidos na teoria de Bayes propuseram outra definição para a quantidade de informação que renomearam como uma medida de "surpresa" (do observador).

De acordo com o subjacente psicológico na teoria em Bayes este efeito é o que provoca a transformação das crenças à priori para as crenças à posteriori. Neste sentido vemos, então, que esta quantidade de informação pode ser medida pela distância -entropia relativa - entre as distribuições à priori e à posteriori do observador sobre os espaços de hipóteses disponíveis.

Chamando de $\mathcal{M}$ o espaço de hipóteses disponíveis com $M \in \mathcal{M}$ esta medida é então relacionada com $\frac{P(D \mid M)}{P(D)}$ de acordo com o teorema de Bayes: $P(M \mid D)=\frac{P(D \mid M)}{P(D)} P(M)$.

Conforme se depreende da equação o efeito de $\mathrm{D}$ é a mudança de $\mathrm{P}(\mathrm{M})$ para $\mathrm{P}(\mathrm{M} \mid \mathrm{D})$.

Isto vale dizer que vemos o dado $\mathrm{D}$ como um operador agindo no espaço de distribuições sobre o espaço dos modelos. Então um dos modos de quantificar a informação, ( medida de surpresa) fornecida por D é a medida da distância , $\log _{2}\left(\frac{\mathrm{P}(\mathrm{D} \mid \mathrm{M})}{\mathrm{P}(\mathrm{D})}\right)$, entre as distribuições à priori e à posteriori.

\section{Probabilidade em escalas temporais}

O cálculo em escalas temporais foi introduzido em 1988 por Stefan Hilger [1], com o intuito de unificar as teorias de sistemas, tanto contínuo quanto discreto.

Uma escala temporal $\mathbb{T}$ é um conjunto fechado não-vazio dos números reais. Exemplos de escalas temporais são $\mathbb{R}, \mathbb{Z},\left\{\frac{1}{2^{k}} ; k \in \mathbb{N}\right\}$, ou o conjunto de Cantor.

Os operadores fundamentais desta teoria unificante são:

$\sigma(\mathrm{t})=\inf \{\mathrm{r} ; \mathrm{r}>\mathrm{t}\} \quad$ - operador de avanço

$\rho(t)=\sup \{r ; r<t\}$ - operador de retardo,

\section{A $\Delta$-derivada é}

$\mathrm{f}^{\Delta}(\mathrm{t})= \begin{cases}\mathrm{f}^{\prime}(\mathrm{t}) & \text { se } \sigma(\mathrm{t})=\mathrm{t} \\ \frac{\mathrm{f}(\sigma(\mathrm{t}))-\mathrm{f}(\mathrm{t})}{\sigma(\mathrm{t})-\mathrm{t}} & \text { se } \sigma(\mathrm{t}) \neq \mathrm{t}\end{cases}$ 
As escalas temporais são próprias para interpretar Probabilidade, pois as variáveis aleatórias e as funções de distribuição podem ser descritas tanto no caso contínuo, como no discreto.

Seja uma escala temporal $\mathbb{T}$ e $A \subset \mathbb{T}$. Então A tem a decomposição $\mathrm{A}=\bigcup_{\mathrm{i}=1}^{\mathrm{m}}\left[\mathrm{a}_{\mathrm{i}}, \mathrm{b}_{\mathrm{i}}\right] \cup\left\{\mathrm{t}_{1}, \mathrm{t}_{2}, \ldots \mathrm{t}_{\mathrm{n}}\right\}$, onde $\tau_{\mathrm{i}}=\sigma\left(\tau_{\mathrm{i}}\right)=\rho\left(\tau_{\mathrm{i}}\right), \quad \forall \tau_{\mathrm{i}} \in\left(\mathrm{a}_{\mathrm{i}}, \mathrm{b}_{\mathrm{i}}\right) \quad$ e $\mathrm{t}_{\mathrm{j}}$-pontos isolados em $\mathbb{T}$.

A $\Delta$-medida de Lebesgue [3], se $\mathrm{A} \neq \varnothing$,é

e $\mu_{\Delta}(\varnothing)=0$.

$$
\mu_{\Delta}(A)=\sum_{i=1}^{m}\left(\sigma\left(b_{i}\right)-a_{i}\right)+\sum_{j=1}^{n}\left(\sigma\left(t_{j}\right)-t_{j}\right)
$$

Definição1: Seja uma escala temporal $\mathbb{T}$ e $\mathfrak{C}_{\mathbb{T}}$ uma álgebra de subconjuntos de $\mathbb{T}$, e $P_{\Delta}$ uma $\Delta$ medida sobre $\mathfrak{C}_{\mathbb{T}}$.Se $P_{\Delta}(\mathbb{T})=1$, então $P_{\Delta}$ é uma medida de probabilidade. Nestas condições a tripla $\left\{\mathbb{T}, \mathfrak{C}_{\mathbb{T}}, P_{\Delta}\right\}$ é chamada um $\Delta$-espaço de probabilidade.

Finalmente podemos definir a $\Delta$-probabilidade de A, assim:

Definição2: Seja $\Omega_{\mathbb{T}}$ um espaço amostral e $A \subset \Omega_{\mathbb{T}}$. Então $\mathrm{P}_{\Delta}(\mathrm{A})=\frac{\mu_{\Delta}(\mathrm{A})}{\mu_{\Delta}\left(\Omega_{\mathrm{T}}\right)}$ é a $\Delta$-probabilidade de A. Como exemplo podemos considerar $\emptyset \neq A \subset \Omega_{\mathbb{T}} \subset \mathbb{N}$. Então $\mathrm{P}_{\Delta}(\mathrm{A})=\frac{\#(\mathrm{~A})}{\#\left(\Omega_{\mathrm{T}}\right)}$, como usualmente.

\section{Objetivo e primeiros resultados}

O objetivo principal deste trabalho é a extensão dos resultados de Baldi e Itti na teoria usando a medida "surpresa" para escalas temporais, e sua aplicação em experimentos de Marketing e em Economia no mercado BOVESPA.

Os primeiros resultados preliminares obtidos de imediato, entre outros, podem ser elencados:

Proposição 1: A probabilidade $P_{\Delta}(K \mid L)=\frac{\mu_{\Delta}(K \cap L)}{\mu_{\Delta}(L)}, \quad$ supondo $\mu_{\Delta}(L) \neq \emptyset$.

Proposição 2: Uma medida de “surpresa” neste contexto $\log _{2}\left(\frac{\mathrm{P}(\mathrm{D} \mid \mathrm{M})}{\mathrm{P}(\mathrm{D})}\right)=\log _{2}\left(\frac{\mu_{\Delta}(\mathrm{M} \cap \mathrm{D}) \mu_{\Delta}\left(\Omega_{\mathrm{T}}\right)}{\mu_{\Delta}(\mathrm{M}) \mu_{\Delta}\left(\mu_{\Delta}(\mathrm{D})\right)}\right)$.

Palavras-chave: Teorema de Bayes, Teoria da Informação, Escalas Temporais, Medida de Surpresa.

\section{Referências}

[1] S. Hilger, Analysis on measure chains - a unified approach to continuous and discrete calculus, Results Math.vol.18,pp.18-56, (1990).

[2] L. Itti \& P. F. Baldi, Bayesian surprise attracts human attention. Vision Research. 49(10), 1295-1306, (2009).

[3] U. Ufuktepe, A. Deniz, Lebesgue-Stieltjes measure on time scales. Turk. J. Math. 32, 1-8 (2009). 